\title{
Quantitative activity-induced manganese-dependent MRI for characterizing cortical layers in the primary somatosensory cortex of the rat
}

\author{
Matthieu Auffret • Idrees Samim • Mario Lepore • \\ Rolf Gruetter • Nathalie Just
}

Received: 11 September 2014/ Accepted: 24 October 2014/Published online: 1 November 2014

(c) Springer-Verlag Berlin Heidelberg 2014

\begin{abstract}
The ability of $\mathrm{Mn}^{2+}$ to follow $\mathrm{Ca}^{2+}$ pathways upon stimulation transform them into remarkable surrogate markers of neuronal activity using activity-induced manganese-dependent MRI (AIM-MRI). In the present study, a precise follow-up of physiological parameters during $\mathrm{MnCl}_{2}$ and mannitol infusions improved the reproducibility of AIM-MRI allowing in-depth evaluation of the technique. Pixel-by-pixel $T_{1}$ data were investigated using histogram distributions in the barrel cortex (BC) and the thalamus before and after $\mathrm{Mn}^{2+}$ infusion, after blood brain barrier opening and after $\mathrm{BC}$ activation. Mean $\mathrm{BC} \mathrm{T}_{1}$ values dropped significantly upon trigeminal nerve (TGN) stimulation $(-38 \%, P=0.02)$ in accordance with previous literature findings. $\mathrm{T}_{1}$ histogram distributions showed that $34 \%$ of $\mathrm{T}_{1 \mathrm{~s}}$ in the range $600-1500 \mathrm{~ms}$ after $\mathrm{Mn}^{2+}+$ mannitol infusions shifted to $50-350 \mathrm{~ms}$ after TGN stimulation corresponding to a twofold increase of the percentage of pixels with the lowest $\mathrm{T}_{1 \mathrm{~s}}$ in $\mathrm{BC}$. Moreover, $\mathrm{T}_{1}$ changes in response to stimulation increased significantly from superficial cortical layers (I-III) to deeper layers (V-VI). Cortical cytoarchitecture detection during a functional paradigm was performed extending the potential of AIM-MRI. Quantitative AIM-MRI could thus offer a means to interpret local neural activity across cortical layers while identification of the role of calcium dynamics in vivo during brain activation could play a key role in resolving neurovascular coupling mechanisms.
\end{abstract}

M. Auffret · I. Samim · M. Lepore · R. Gruetter · N. Just ( $\square)$ Laboratory for Functional and Metabolic Imaging (LIFMET), Centre d'Imagerie Biomédicale-Animal Imaging and

Technology Core (CIBM-AIT), Ecole Polytechnique Fédérale de Lausanne, CH-1015 Lausanne, Switzerland

e-mail: nathalie.just@epfl.ch
Keywords Manganese - Histograms - Barrel cortex · Thalamus $\cdot$ Cortical layers

\section{Introduction}

Manganese-enhanced MRI (MEMRI) is a powerful technique allowing an enhanced definition of structural, anatomical, and functional details in the rodent's brain (Silva et al. 2008; Tucciarone et al. 2009; Just and Gruetter 2011; Koretsky and Silva 2004; van der Linden et al. 2004; Inoue et al. 2011; Serrano et al. 2008). Systemic administration of $\mathrm{Mn}^{2+}$ several hours before image acquisition (24-72 h) has proved to be a unique strategy to investigate the architecture of specific brain areas with MRI (Silva et al. 2008; Tucciarone et al. 2009). Manganese can also be used in different ways for functional investigations. Among the possibilities, $\mathrm{Mn}^{2+}$ can act as an in vivo trans-synaptic tract tracer allowing the investigation of either connectivity or axonal transport (Inoue et al. 2011). None of these functional strategies necessitate blood brain barrier (BBB) permeabilization but require injection of $\mathrm{Mn}^{2+}$ several hours before MR image acquisition. Tucciarone (2009) and Yu et al. 2012a, b demonstrated for example that MEMRI allowed the direct visualization of thalamo-cortical inputs to the barrel cortex and plasticity changes (Yu et al. 2012a) as well as the depiction of cortical layers (Lu et al. 2004; Silva et al. 2008; Tucciarone et al. 2009; Yu et al. 2012a, b, 2014). 3D MRI techniques have been used allowing increased spatial resolution of the brain architecture and of the sensory pathways to specific areas as well as a better investigation of connected areas.

In 1997, Lin et al. (Lin and Koretsky 1997) proposed an alternative to blood oxygen level-dependent (BOLD) functional MRI to image brain function: $\mathrm{Mn}^{2+}$ allowed for 
the first time the indirect measurement of neuronal activation within a few minutes through activity-induced manganese-dependent (AIM) MRI. The paramagnetic properties of $\mathrm{Mn}^{2+}$ and their ability to follow $\mathrm{Ca}^{2+}$ ion pathways upon stimulation/depolarization due to their analogous features transformed them into remarkable surrogate markers of neuronal activity (Just and Gruetter 2011; Just et al. 2011b; Chuang et al. 2009; Silva et al. 2008; Tucciarone et al. 2009). The latter technique represents a third possibility for assessing function in the brain of rodents and requires a disrupted BBB. The co-localization of BOLD, cerebral blood flow (CBF), and manganese uptake responses proved that AIM-MRI measured calcium-dependent synaptic activity (Duong et al. 2000). Despite the fact that AIM-MRI remains difficult due essentially to acute toxicity effects of $\mathrm{Mn}^{2+}$ and the disruption of the BBB (see Table 1) (Aoki et al. 2004; Weng et al. 2007; Pautler 2006), it is a unique technique that provides high sensitivity and high signal to noise ratio compared to BOLD fMRI techniques.

During neuronal activity due to somatosensory stimulation, $\mathrm{Ca}^{2+}$ influx through voltage-gated (dependent) $\mathrm{Ca}^{2+}$ channels results in a transient increase of intracellular $\mathrm{Ca}^{2+}$ (Lauritzen 2005; Schulz et al. 2012). These changes have shown to be of great interest for understanding the link between neuronal activity and hemodynamic response in BOLD fMRI (Lauritzen 2005; Schulz et al. 2012; Lecrux et al. 2011). The investigation of calcium dynamics is of paramount importance and has been the topic of many research projects interested in the role of $\mathrm{Ca}^{2+}$ transients across cortical layers in the rodent barrel cortex (Svoboda et al. 1999; Helmchen et al. 1999; Egger et al. 1999; Stosiek et al. 2003; Wang et al. 2006; Benedetti et al. 2011). Most of these studies were performed with optical imaging techniques using ex vivo samples. Although fiber-optic calcium recording was performed within an MRI scanner allowing simultaneous BOLD fMRI (Schulz et al. 2012), the technique remains demanding and spatially limited. The potential of AIM-MRI, on the other end, has not yet been fully evaluated and remains poorly quantified, based on $\mathrm{T}_{1}$ mapping and average values.
The purpose of the present study was to develop the use of histogram analysis of $T_{1}$ distributions within the rat barrel cortex and the thalamus for an improved quantitative outcome of AIM-MRI and to investigate $\mathrm{T}_{1}$ responses to accumulation of $\mathrm{Mn}^{2+}$ in cortical layers of the barrel cortex following Trigeminal nerve (TGN) stimulation.

\section{Materials and methods}

\section{Animal preparation}

All animal procedures were performed according to the Swiss federal guidelines of the Animal Care and approved by the local authority. As outlined in Table 2, 15 SpragueDawley rats (SD) were initially prepared as described by Lin and Koretsky (1997) and Duong et al. (2000). The low survival rate of this group of rats $\left(8 / 15\right.$ died during $\mathrm{MnCl}_{2}$ infusion, 4/15 died at mannitol injection and 3/15 during surgery) prompted us to change our protocol. Surgery, carotid artery catheterization, and mannitol injections were tested during bench top experiments on $10 \mathrm{SD}$ rats as described below and after injection of $1 \%$ Evans Blue (EB) to assess blood barrier breakage were rapidly sacrificed and their brain collected. In the present work, we essentially report research on male adult SD rats $(n=10$, $350 \pm 40 \mathrm{~g}$ ) for which procedures were validated in prior experiments. These rats were initially anesthetized with isoflurane (4 \% ISOCARE, Animalcare LTD, York, UK) in a mixture of $\mathrm{O}_{2}$. Each rat was orally intubated. Two femoral arteries and femoral veins were catheterized for blood gas sampling and blood pressure monitoring as well as for $\alpha$-chloralose (Bolus of $80 \mathrm{mg} / \mathrm{kg}$ followed by infusion at $27 \mathrm{mg} / \mathrm{kg} / \mathrm{h}$ ) and $\mathrm{MnCl}_{2}$ administrations. Pancuronium Bromide (Pavulon ${ }^{\circledR}$ ) was injected at a dose of $0.7 \mathrm{ml} / \mathrm{h} /$ $350 \mathrm{~g}$ to avoid spurious motion and tremors.

\section{Surgical procedure}

Each rat was anesthetized 3 min with $4 \%$ isoflurane, which was switched to $2 \%$ and the ventral neck region

Table 1 Activity-induced manganese MRI studies

\begin{tabular}{|c|c|c|c|c|}
\hline Study & Dose $\mathrm{MnCl}_{2} /$ rate of infusion & Number of animals & Anesthesia & Stimulation procedure \\
\hline Lin and Koretsky (1997) & $120 \mathrm{mM}(3.6 \mu \mathrm{mol} / \mathrm{min} \mathrm{i} . \mathrm{v})$ & 5 & 1-1.8\% Halothane & Glutamate and forepaw \\
\hline Duong et al. (2000) & $120 \mathrm{mM}(2 \mathrm{~mL} / \mathrm{h}$ i.v $)$ & 7 & Alpha-Chloralose & Forepaw stimulation \\
\hline Aoki et al. (2002) & $74.5 \mathrm{mM}(3.2 \mathrm{ml} / \mathrm{h})$ & 5 (Glutamate $)+5$ (forepaw) & Alpha-Chloralose + urethane & Glutamate and forepaw \\
\hline Weng et al. (2007) & 128 mM (2 ml/IP) & 6 & Sodium pentobarbital & Whisker stimulation \\
\hline Zhang et al. (2014) & $50 \mathrm{mM}(1.8 \mathrm{ml} / \mathrm{min}$ i.a) & 6 & Urethane & Visual stimulation \\
\hline Fa et al. (2011) & $25 \mathrm{mM}(1.8 \mathrm{ml} / \mathrm{min}$ i.a $)$ & 12 (6 per group) & $2 \%$ Isoflurane & Visual stimulation \\
\hline
\end{tabular}


Table 2 Summary of experiments performed in the present study

\begin{tabular}{|c|c|c|c|c|c|}
\hline Study & $\begin{array}{l}\text { Dose } \mathrm{MnCl}_{2} / \text { rate } \\
\text { of infusion }\end{array}$ & $\begin{array}{l}\text { Number of } \\
\text { animals }\end{array}$ & Anesthesia & $\begin{array}{l}\text { Stimulation } \\
\text { procedure }\end{array}$ & Reason for Death \\
\hline $\begin{array}{l}\text { Protocol following Lin and } \\
\text { Koretsky (1997) and Duong } \\
\text { et al. (2000) }\end{array}$ & $120 \mathrm{mM}(3.6 \mu \mathrm{mol} / \mathrm{min}$ i.v $)$ & 15 & Alpha-Chloralose & None & $\begin{array}{l}\text { Toxicity of } \mathrm{MnCl}_{2}(8) \\
\text { and mannitol (3) Surgery (4) }\end{array}$ \\
\hline $\begin{array}{l}\text { In-house protocol with a T } \\
\text { shape catheter follow-up } \\
\text { of Blood Pressure Heart rate }\end{array}$ & $\begin{array}{l}25 \mathrm{mM}(1.5 \mu \mathrm{mol} / \mathrm{min} \\
\text { to } 6.5 \mu \mathrm{mol} / \mathrm{min}) \mathrm{I} . \mathrm{V}\end{array}$ & 10 & Alpha-Chloralose & Whisker stimulation & None \\
\hline
\end{tabular}

were shaved from the chin down to just above the sternum. A straight cut of the skin was made starting immediately below the chin all the way to the top of the sternum just above the ribcage.

The carotid vasculature is located in a fat cushion in depth on the sternohyoid muscle. The dissection continued to expose carotid bifurcation into the internal and external branches while taking care of preserving the vagus nerve.

The external carotid artery was ligated with suture. The common carotid artery was exposed. A homemade T-shaped catheter was inserted at the extremities of the common carotid artery. The blood was allowed to circulate in the catheter loop restoring carotid artery circulation. The wound was closed with suture looping suture around the tubes of the catheter so to have them fixed to the skin.

$\mathrm{MnCl}_{2}$ and mannitol administration

A manganese chloride isotonic solution at $100 \mathrm{mM}$ (Sigma-Aldrich, Switzerland, Molecular weight: $124.5 \mathrm{~g} /$ mol) was prepared using pure water. The $100 \mathrm{mM}$ standard solution was diluted to $25 \mathrm{mM}$ using $0.9 \%$ sodium chloride. The solution was constantly infused into the rat femoral vein with a syringe pump at starting rate at $3.6 \mathrm{ml} /$ h. During infusion, the blood pressure was carefully followed and if the blood pressure dropped (or increased) by more than $10 \%$, the infusion process was stopped until the animal physiology was stable again and blood pressure parameters were returned to their initial values. If the blood pressure stayed stable during a $500 \mu \mathrm{l}$ infusion of $\mathrm{MnCl}_{2}$ then the rate of infusion was increased by $2 \mathrm{ml} / \mathrm{h}$ up to a maximum rate of $18 \mathrm{ml} / \mathrm{h}$. If the blood pressure was systematically decreased by more than $10 \%$ at a specific rate of infusion even when infusion had been stopped, the rate at which blood pressure was not affected was chosen and infusion was pursued across a longer period until $8 \mathrm{ml}$ of $\mathrm{MnCl}_{2}$ were infused.

A solution of $25 \%$ D-Mannitol (Sigma-Aldrich, Switzerland) was prepared and manually injected through the loop catheter inserted in the internal carotid artery $(3 \mathrm{ml} /$ $300 \mathrm{~g}$ ). Injection was slow and care was taken to pinch the catheter part inserted in the common carotid artery so that no mannitol goes to the heart. For the same reasons, the loop catheter was not flushed after mannitol injection. The mannitol injection was performed with the rat holder outside of the magnet and while again following carefully the breath rate and the blood pressure known to increase after mannitol injection. No $\mathrm{MnCl}_{2}$ was infused during this manipulation. The rat holder was placed back at the same position in the magnet. As the BBB remains permeable to manganese for a period ranging from 5 to $35 \mathrm{~min}$, care was taken to proceed rapidly for further scanning procedures.

Trigeminal nerve stimulation

Electrical stimulation of the TGN contralateral to the BBB leakage was performed by delivering square pulses using an external stimulator (WPI, Stevenage, UK) (Just et al. 2010). The cathode was inserted in the hiatus infraorbitalis as described in (Norup Nielsen and Lauritzen 2001) and the anode was inserted either in the masticatory muscles. Pulses with a $0.5 \mathrm{~ms}$ width were delivered every second $(1 \mathrm{~Hz})$ at a current intensity of $2 \mathrm{~mA}$ during $10 \mathrm{~min}$.

\section{MRI and data analysis}

All the experiments were performed on an actively shielded 9.4T/31 cm bore magnet (Magnex, Varian, Abingdon, UK) with $12 \mathrm{~cm}$ gradients $(400 \mathrm{mT} / \mathrm{m}$ in $120 \mu \mathrm{s})$ with a quadrature Transmit/Receive $17 \mathrm{~mm}$ surface coil. Animals were anesthetized with $\alpha$-chloralose and set in an MRcompatible MR cradle. Their heads were secured in a custom-made stereotaxic frame consisting of ear pieces and a bite bar. Rectal temperature and blood gases were monitored and maintained at $T=37.5-38{ }^{\circ} \mathrm{C}$, Partial Pressure of $\mathrm{CO}_{2}\left(\mathrm{PCO}_{2}\right)=38-45 \mathrm{mmHg}$, mean arterial blood pressure $(\mathrm{MABP})=150-180 \mathrm{mmHg}, \quad \mathrm{pH}=7.3 \pm 0.1$, Heart Rate $(\mathrm{HR})=300-500$ Beats per minutes $(\mathrm{BPM})$. First- and second-order shims were adjusted using fast, automatic shimming technique by mapping along projections (FASTMAP) (Mlynárik et al. 2006) resulting in water linewidths of $11-15 \mathrm{~Hz}$ in a $216 \mu \mathrm{l}\left(6 \times 8 \times 4.5 \mathrm{~mm}^{3}\right)$ volume. $3 \mathrm{D}$ gradient-echo $\mathrm{MR}$ images were acquired before manganese infusion, before mannitol injection, after 


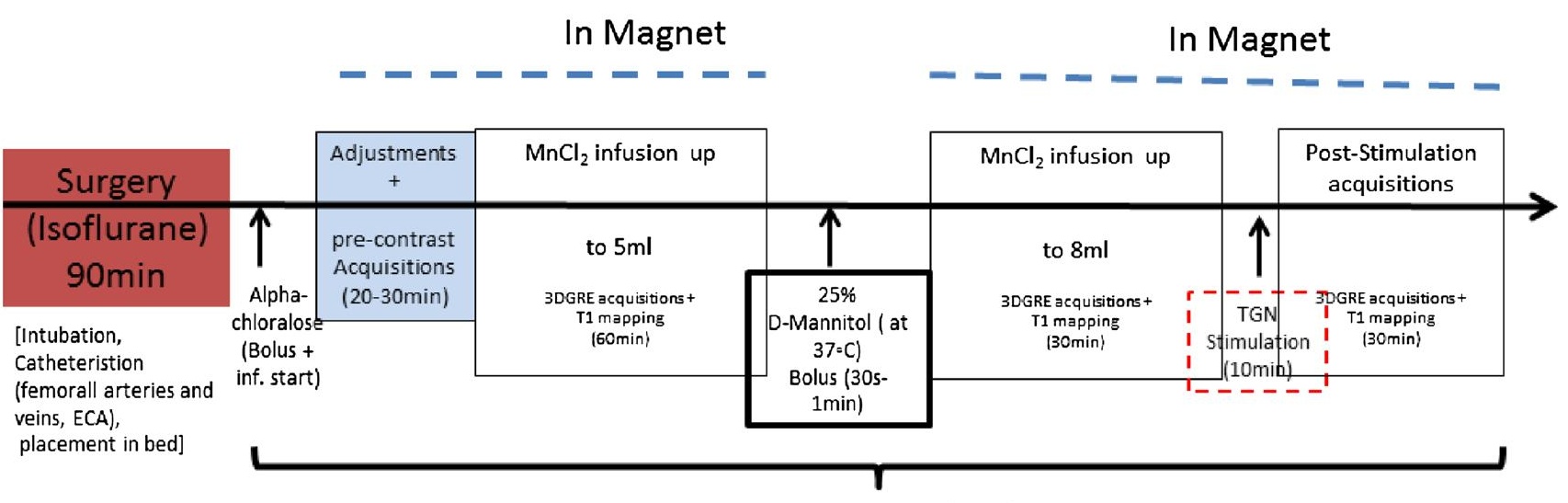

Monitoring temperature, $\mathrm{PCO} 2, \mathrm{PO} 2$, Blood Pressure, Heart Rate

Fig. 1 Timeline of experiments

BBB breakage with mannitol and after TGN stimulation $\left(\mathrm{TR} / \mathrm{TE}=23 / 4 \mathrm{~ms}\right.$, Flip angle $=90^{\circ}, \mathrm{FOV}=21 \times 21 \times$ $21 \mathrm{~mm}^{3}$, matrix size $=128 \times 128 \times 32$ or $64 \times 64 \times 32$, coronal slices, $\mathrm{BW}=24 \mathrm{kHz}, 3-4$ averages). T1 mapping Saturation Recovery TURBOFLASH (TR/TE $=200 /$ $3.1 \mathrm{~ms}$, flip angle $=45^{\circ}$, Recovery time (TSR), TSR $=0.02-14 \mathrm{~s}, \quad$ step $=0.5 \mathrm{~s}, \quad 2-6$ slices, $\quad$ FOV $=$ $21 \times 21 \mathrm{~mm}^{2}$, Matrix size $=64 \times 64$ or $128 \times 128$, Thickness $(\mathrm{TH})=1 \mathrm{~mm}$ images, Acquisition time: 15-30 min) (Parker et al. 2000) were reconstructed using in-house written Matlab routines. The timeline of experiments is given in Fig. 1.

Magnetic resonance images were processed and analyzed using Image processing software (Image J 1.3.1_13. NIH, USA) as well as custom written routines running in Matlab (Matlab R2010, The MathWorks, USA). T1 maps were constructed on a pixel-by-pixel basis. For cross-subject analysis and comparisons of contrasts before and after blood brain breakage, $\mathrm{T}_{1}$ maps were thresholded. ROIs of identical volumes were drawn in the rat barrel cortex and the thalamus. These areas covered the barrel cortex somatosensory cortex and thalamic nuclei encompassing the ventral posterior medial nucleus (VPM) and posterior medial nucleus (POM) with respect to the Paxinos and Watson atlas (Paxinos and Watson 1998).

Histogram analysis (Just 2011c) was performed for each rat and each ROI. Data were normalized to the total number of pixels within each ROI. For comparison purposes, bins of identical size were chosen. Descriptive analysis of histograms was performed with ORIGIN (ORIGINLAB 9.0, Germany).

\section{Statistics}

Unless otherwise stated, all summary data were presented as mean $\pm \mathrm{SD}$. Statistical analyses were carried out using a
One-way ANOVA test with Bonferroni correction. A $p<0.05$ was considered significant.

\section{Results}

Blood brain barrier breakage and physiology during $\mathrm{Mn}^{2+}$ infusion

In Fig. 2a, b, blood pressure (BP) and heart rate (HR) evolutions are shown for a representative rat that died after infusing $2.4 \mathrm{ml}$ of $\mathrm{MnCl}_{2}$ at $120 \mathrm{mM}$. The heart rate dropped significantly after 25 min of infusion at a slow rate $(3.5-4.5 \mathrm{ml} / \mathrm{h})$. Figure $2 \mathrm{c}$ shows both mean $\mathrm{BP}$ and $\mathrm{HR}$ (eight rats) as a function of time during $\mathrm{MnCl}_{2}$ infusion (120 mM). Both BP and HR dropped significantly after 40 min of $\mathrm{MnCl}_{2}$ infusion. On individual rats, BP could be controlled easily and increased back to initial levels (as indicated by arrows on Fig. 2a). However, HR appeared to be a critical parameter indicating definitive loss of physiological conditions as soon as it dropped $(<10 \%)$.

When monitoring HR carefully, the survival of rats was increased compared to our preliminary studies. Each time blood pressure dropped by more than $10 \%$, the infusion was stopped and restarted when all the parameters were stabilized. Figure $2 \mathrm{~d}$ shows the evolution of HR (BPM) during the infusion of $\mathrm{MnCl}_{2}(25 \mathrm{mM})$ at an evolving rate $(\mathrm{ml} / \mathrm{h})$ in a representative rat: the rate of infusion was increased up to $15.6 \mathrm{ml} / \mathrm{h}$. The rat survived and underwent Mannitol injection and TGN stimulation. The corresponding evolution of BP (mmHg) is also shown in Fig. 2e. Figure $2 \mathrm{f}$ shows both stable mean $\mathrm{BP}$ and $\mathrm{HR}$ (ten rats) as a function of time during $\mathrm{MnCl}_{2}(25 \mathrm{mM})$. The carefully monitored physiology allowed repeated and reproducible investigations in ten rats that survived through the TGN stimulation. 
B

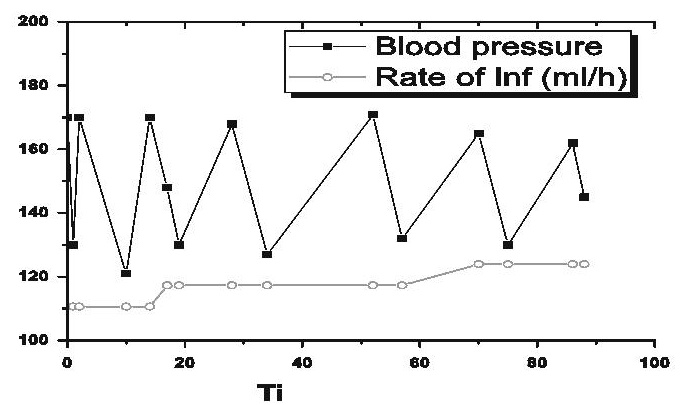

C

The protocol for BBB breakage with mannitol injection in the internal carotid artery was tested on animals outside the magnet. Ten rats received a $1 \mathrm{ml}$ injection of Evans Blue (EB, $1 \%$ ) solution 1 min after injection of mannitol. The assessment of the BBB integrity using EB is widely accepted as it only leaks to the brain parenchyma when the BBB is either absent, immature, or significantly disrupted. After sacrifice, the brain was quickly removed and demonstrated mild to strong staining of the BBB broken part of the brain on eight animals. An example on a representative animal on the whole brain (Fig. 3a) and on axial sections (Fig. 3b) is shown.
E

$\mathbf{F}$
Evaluation of AIM-MRI and mean $\mathrm{T}_{1}$ values in the barrel cortex and the thalamus

Figure $3 \mathrm{c}$ are 3D-GRE MR $\mathrm{T}_{1}$-weighted images of a representative rat brain before and after $\mathrm{MnCl}_{2}$ infusion. After injection of Mannitol, the BBB was broken and $\mathrm{MnCl}_{2}$ accumulated into the thalamus and cortical areas. Upon 10 min TGN stimulation, $\mathrm{MnCl}_{2}$ further accumulated into the barrel cortex. 3D-GRE MR $\mathrm{T}_{1}$-weighted images (Fig. 3d) of consecutive axial slices resulting from subtraction of post-stimulation images (Fig. 3f) and pre-stimulation images (Fig. 3e) were obtained allowing the 

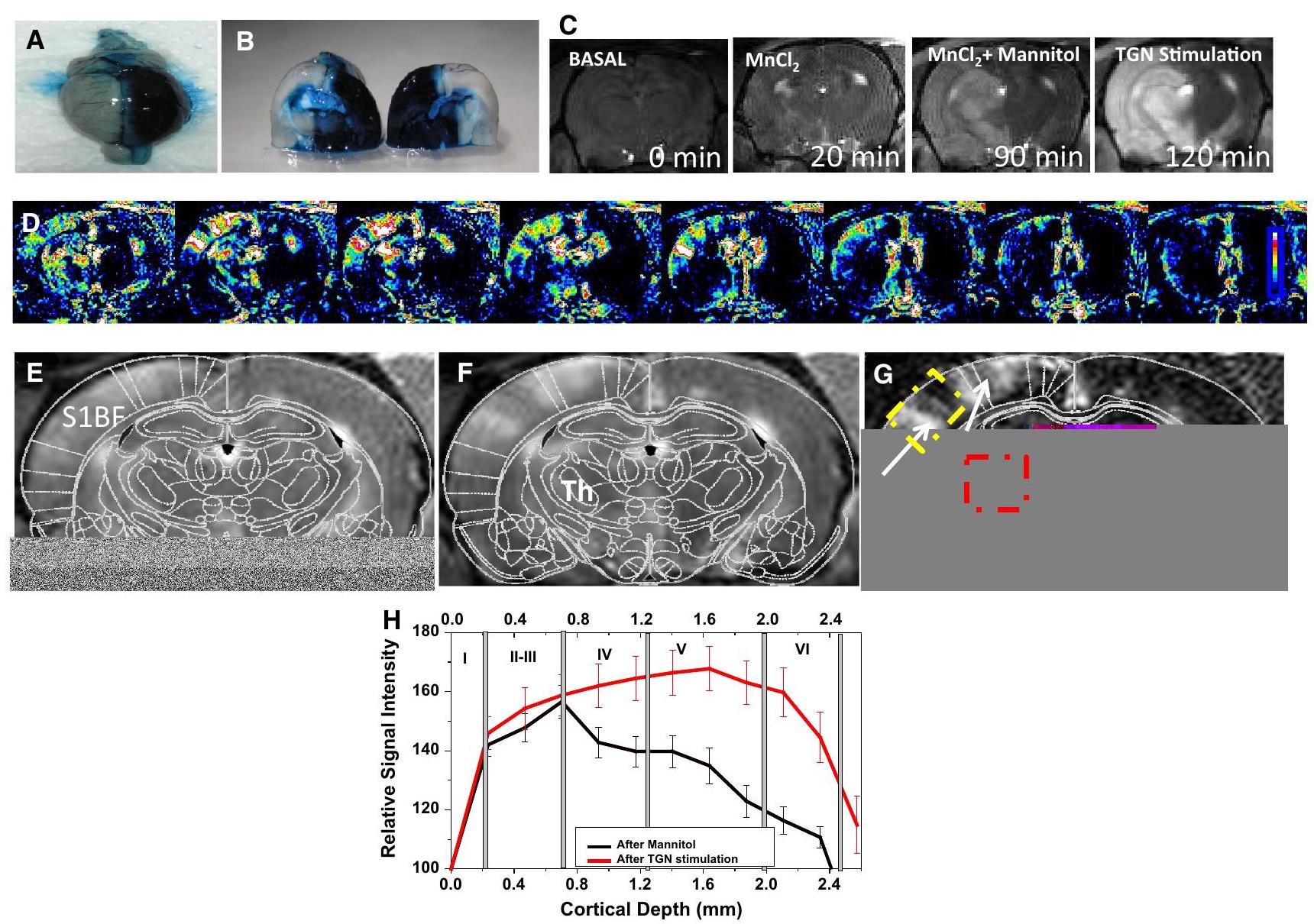

Fig. 3 a, b Evans Blue staining after injection of $25 \%$-D-Mannitol to assess the integrity of the blood brain barrier. a Whole brain and b axial sections. c Dynamic activity-induced manganese MRI. Examples of 3D gradient-echo T1-weighted MR Magnitude images $\left(\mathrm{TR} / \mathrm{TE}=23 / 4 \mathrm{~ms}\right.$, Flip angle $=90^{\circ}, \mathrm{FOV}=21 \times 21 \times 21 \mathrm{~mm}^{3}$, matrix size $=128 \times 128 \times 32$, coronal slices, $\mathrm{BW}=24 \mathrm{kHz}, 3-4$ averages). Increased intensity can be seen in the cortex and in thalamic areas. d False Color-coded difference images (3D-GRE; TGN stimulation post-mannitol) of consecutive slices in a representative rat demonstrating strong activation in cortical and thalamic areas contralateral to the stimulation. e-g 3D Gradient-Echo

visualization of accumulation of $\mathrm{Mn}^{2+}$ in thalamic nuclei and in cortical areas upon TGN stimulation. Overlaying the Paxinos and Watson atlas onto these images allowed defining ROIs for further $\mathrm{T}_{1}$ analysis (Fig. $3 \mathrm{~g}$ ). The AIMMRI relative signal intensities (averaged across rats) after Mannitol and after TGN stimulation were drawn as a function of cortical depth (Fig. 3h) demonstrating a stronger and significant enhancement from layer IV to layer VI during stimulation compared to post-mannitol step.

$\mathrm{T}_{1}$ values were evaluated on a pixel-by-pixel basis and over manually drawn ROIs in the thalamus and the primary somatosensory cortex (Fig. 4a) before $\mathrm{MnCl}_{2}$ infusion, magnitude images before and after TGN stimulation with Paxinos atlas overlay. g Difference image with Paxinos atlas and arrows indicating $\mathrm{Mn}^{2+}$ enhancement in the primary somatosensory barrel cortex (S1BF) and the thalamus (Th). Region of interest for further $\mathrm{T}_{1}$ analysis are shown. h Average $( \pm$ SEM) signal intensity profiles across the cortex as a function of the cortical depth for $n=10$ rats after Mannitol injection (90 min, black curve) and after TGN stimulation (110 min, red curve). A significant difference was observed in cortical layers IV-V and VI after TGN stimulation compared to the mannitol step ( $p<0.05$, error bars \pm SEM)

after $\mathrm{MnCl}_{2}$ and Mannitol infusions and after TGN stimulation using a heavily T1-weighted SRTF sequence. In Fig. $4 \mathrm{~b}$, thresholds were defined for $\mathrm{T}_{1}$ maps at a lower $\mathrm{T}_{1}$ $(0.8 \mathrm{~s})$ allowing an improved depiction of $\mathrm{T}_{1}$ changes in $\mathrm{BC}$ and $\mathrm{Th}$ as indicated by arrows. Mean $\mathrm{T}_{1}$ values across the different conditions are presented in Fig. 4c. Before $\mathrm{MnCl}_{2}$ infusion, mean $\mathrm{T}_{1}$ values in the barrel cortex and the thalamus ranged between 1.7 and $2 \mathrm{~s}$, respectively, and there were no significant difference between the left and right hemispheres. After Mannitol injection through the internal carotid artery, mean $\mathrm{T}_{1}$ values dropped significantly ( $-43 \%$, one-way ANOVA, $P=0.005)$ in the barrel cortex and the thalamus in both hemispheres. A 

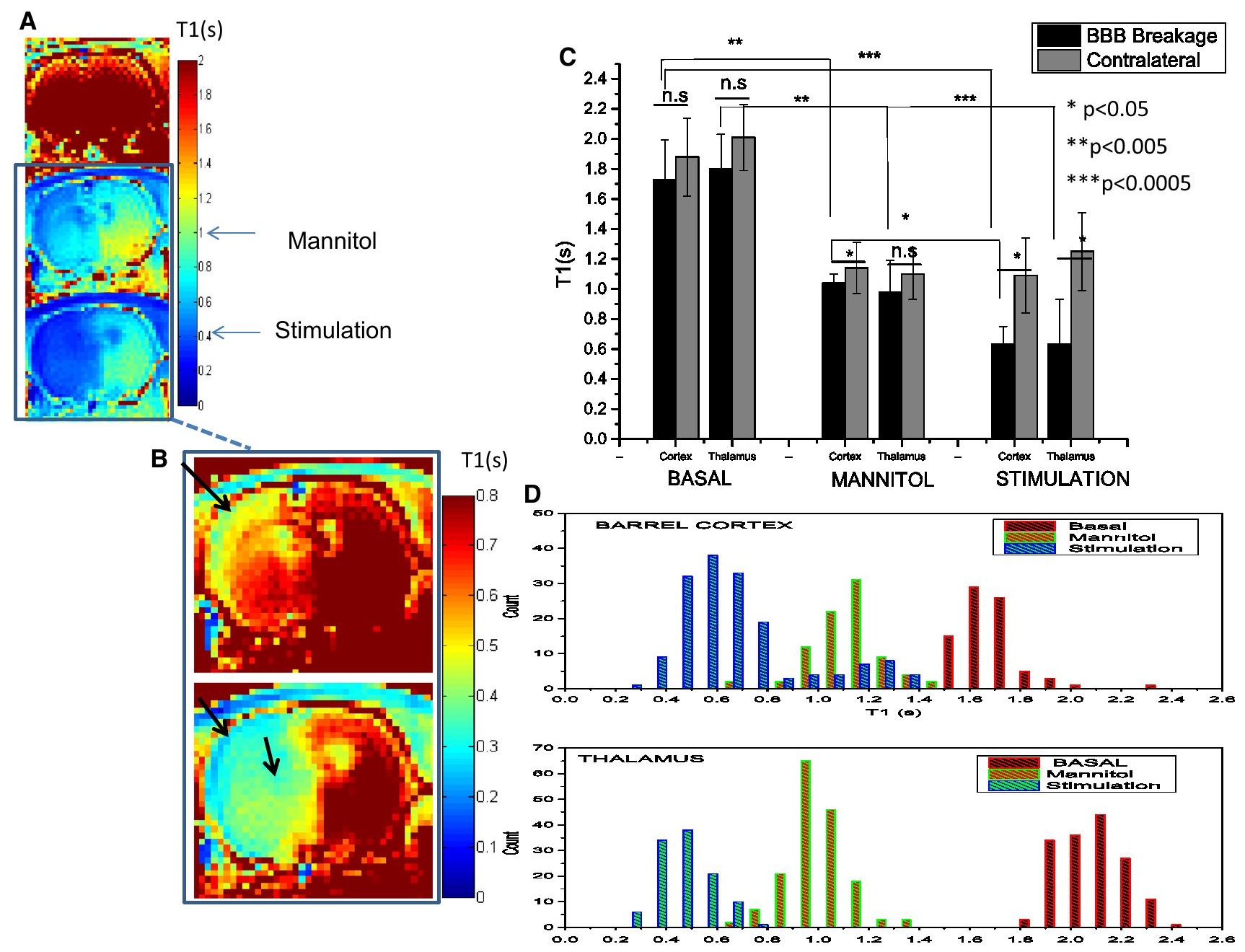

Fig. $4 \mathrm{~T}_{1}$ mapping. a $\mathrm{T}_{1}$ maps obtained on a pixel-by-pixel basis using an SRTF sequence. $\left(\mathrm{TR} / \mathrm{TE}=200 / 3.1 \mathrm{~ms}\right.$, flip angle $=45^{\circ}$, recovery time (TSR), TSR $=0.02-14 \mathrm{~s}$, step $=0.5 \mathrm{~s}, 2-6$ slices, FOV $=21 \times 21 \mathrm{~mm}^{2}$, Matrix size $=64 \times 64$ or $128 \times 128$, $\mathrm{TH}=1 \mathrm{~mm}$ images) (Parker et al. 2000). From top to bottom: $\mathrm{T}_{1}$ map before $\mathrm{Mn}^{2+}$ infusion, after $\mathrm{Mn}^{2+}$ and mannitol infusions leading to BBB breakage of half side of the brain and during 10-minute TGN stimulation. $\mathbf{b}$ Thresholds for $\mathrm{T}_{1}$ maps after BBB breakage and during stimulation for an increased visualization of $\mathrm{T}_{1}$ changes in SIBF and in the thalamus (arrows). c Mean $\mathrm{T}_{1}$ values across the different conditions. Before $\mathrm{MnCl}_{2}$ infusion, mean $\mathrm{T}_{1}$ values in the barrel cortex and the thalamus presented no significant (n.s) difference between the left and right hemispheres. After Mannitol injection, mean $\mathrm{T}_{1}$ values dropped significantly ( $-43 \%$, one-way ANOVA,

significant difference in the barrel cortex of the BBB broken side (ANOVA, $P=0.015$ ) was found compared to the contralateral side but not in the thalamus. Upon TGN stimulation, mean $\mathrm{T}_{1}$ values in the barrel cortex of the BBB broken side further dropped demonstrating a significant difference compared to the mannitol stage ( $-38 \%$, oneway ANOVA, $P=0.02$ in barrel cortex). In the thalamus, mean $\mathrm{T}_{1}$ decrease upon TGN stimulation demonstrated a strong tendency to decrease ( $P=0.06$ in thalamus).
** $P=0.005)$ in the barrel cortex and the thalamus in both hemispheres. Upon TGN stimulation, mean $\mathrm{T}_{1}$ values in the barrel cortex of the $\mathrm{BBB}$ broken side further dropped demonstrating a significant difference compared to the mannitol stage ( $-38 \%$, oneway ANOVA, $* P=0.02)$ A strong tendency for decreased $\mathrm{T}_{1}$ was observed in the thalamus. ( $P=0.06$ in thalamus). d Histogram analysis of $\mathrm{T}_{1}$ distributions in the barrel cortex and the thalamus in a representative animal: $\mathrm{T}_{1}$ distributions within each ROI of the $\mathrm{BBB}$ broken side in the basal condition, after $\left(\mathrm{Mn}^{2+}+\right.$ mannitol) infusions and after TGN stimulation (a). Within the barrel cortex, the number of pixels with $\mathrm{T}_{1 \mathrm{~s}}$ in the range $0.2-0.6 \mathrm{~s}$ was multiplied by 2 after the stimulation. In the thalamus, there was also a shift of $\mathrm{T}_{1}$ values in the range $1-1.5 \mathrm{~s}$ toward the range $0.25-0.5 \mathrm{~s}$

Histogram analysis of $\mathrm{T}_{1}$ distributions in the barrel cortex and thalamus

The $\mathrm{T}_{1}$ distributions over each $\mathrm{ROI}$ were compared showing a significant shift of $\mathrm{T}_{1}$ values in the range $1.2-3.0 \mathrm{~s}$ in the basal condition to $0.6-1.5 \mathrm{~s}$ after $\left(\mathrm{MnCl}_{2}+\right.$ Mannitol $)$ and $0.05-0.75 \mathrm{~s}$ after stimulation whether in the somatosensory cortex or the thalamus (Fig. 4d) for a representative animal. 

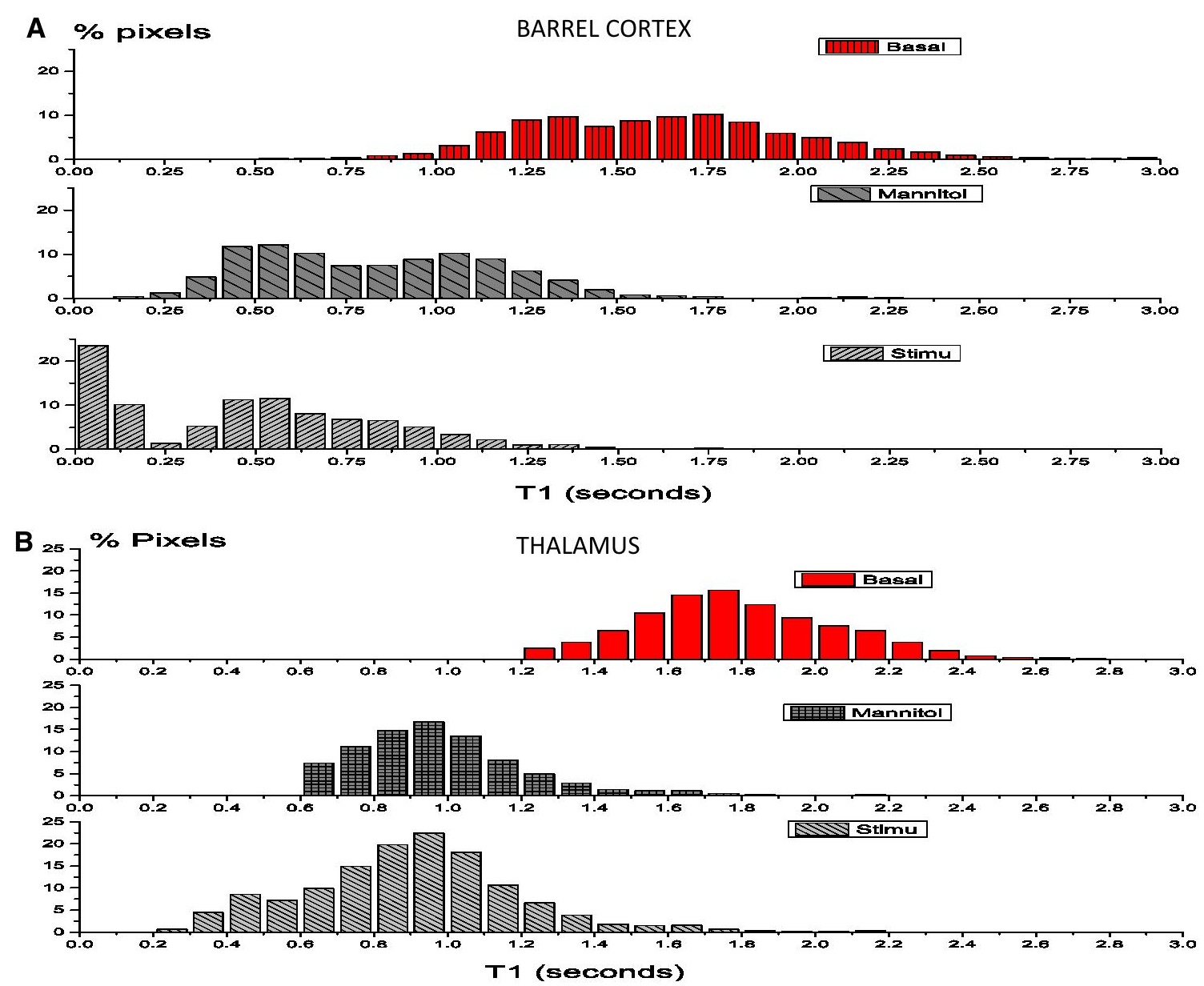

Fig. 5 a Pooled $T_{1}$ distributions for all the animals within the barrel cortex, the percentage of pixels with $\mathrm{T}_{1 \mathrm{~s}}$ in the range $0.05-0.75 \mathrm{~s}$ was multiplied by 1.8 after the stimulation step. $\mathbf{b}$ Pooled $\mathrm{T}_{1}$ distributions

$\mathrm{T}_{1}$ distributions for all the animals were pooled together demonstrating identical trends: within the barrel cortex, the percentage of pixels with $\mathrm{T}_{1 \mathrm{~s}}$ in the range $0.05-0.75 \mathrm{~s}$ was multiplied by 1.8 after the stimulation step (Fig. 5a). In the thalamus, there was also a shift of $\mathrm{T}_{1}$ values in the range $0.2-0.75 \mathrm{~s}$ with 1.5 more pixels after the stimulation step (Fig. 5b). $71 \%$ of the pixels encompassed in the ROI drawn in the barrel cortex had lower $\mathrm{T}_{1}$ values $(150-1150 \mathrm{~s})$ due to BBB breakage and accumulation of $\mathrm{MnCl}_{2}$ in the barrel cortex. Due to TGN stimulation $33.85 \%$ of pixels in the $\mathrm{T}_{1}$ range 600-1500 $\mathrm{ms}$ after mannitol injection had shifted to lowest $\mathrm{T}_{1}$ values in the range $50-350 \mathrm{~ms}$.

Descriptive analyses of the $\mathrm{T}_{1}$ distributions in the barrel cortex and the thalamus were performed for the basal (B), the mannitol (M) and the stimulation (ST) stages of each rat. Quantitative results are presented in Table 3. TGN stimulation induced fewer changes in $\mathrm{T}_{1}$ than $\mathrm{Mn}^{2+}$ accumulation in Th and BC following BBB breakage (Th: 41 vs. $48 \%$; $\mathrm{BC}$ : 34 vs. $49 \%$ ). $\mathrm{T}_{1}$ changes were the largest for the 25 th percentile (T1 dropped by $81 \%$ in $\mathrm{BC}$, and for all the animals within the thalamus, there was also a shift of $\mathrm{T}_{1}$ values in the range $0.2-0.75 \mathrm{~s}$ with 1.5 more pixels after the stimulation step

$78 \%$ in Th due to TGN stimulation). The skewness (S) and kurtosis (K) parameters represent measures of the asymmetry and the peakedness of the distributions, respectively. In the barrel cortex, $\mathrm{T}_{1}$ distributions were all positively skewed while kurtosis increased from basal to stimulation (B: $K=11.9 ; \mathrm{M}: K=19.4 ; \mathrm{ST}: K=21.2$ ) showing an important peak for the lowest $\mathrm{T}_{1}$ values. In the thalamus, the basal $\mathrm{T}_{1}$ distribution was almost normal $(S=0.03$; $K=0.55)$. After mannitol injection, the $\mathrm{T}_{1}$ distribution was positively skewed with a fat right tail $(S=0.46$; $K=1.03)$ while for stimulation, the $\mathrm{T}_{1}$ distribution became flatter with a fat left tail $(S=0.26 ; K=-1.065)$.

Evaluation of $\mathrm{Mn}^{2+}$ accumulation across cortical layers of the barrel cortex and thalamocortical inputs

Difference images were obtained after subtracting 3D-GRE images acquired after TGN stimulation and images postmannitol infusion as shown in six successive false colorcoded slices in Fig. 6a. Cortical layers could be depicted as 
Table 3 Descriptive analysis of T1 histograms in the thalamus and the barrel cortex

\begin{tabular}{lllllllcc}
\hline Thalamus & Mean T1 (s) & SD & Skewness & Kurtosis & Minimum T1 (s) & $\begin{array}{l}\text { 25\% Percentile } \\
\text { T1 (s) }\end{array}$ & $\begin{array}{l}\text { Median } \\
\text { T1 (s) }\end{array}$ & $\begin{array}{l}75 \% \text { Percentile } \\
\text { T1 (s) }\end{array}$ \\
\hline BASAL (B) & 1.8 & 0.3 & 0.0 & 0.6 & 0.7 & 1.6 & 1.8 & 2.0 \\
Mannitol (M) & 0.9 & 0.3 & 0.5 & 1.0 & 0.2 & 0.7 & 0.9 & 1.1 \\
Stimulation (ST) & 0.6 & 0.4 & 0.3 & -1.1 & 0.1 & 0.2 & 0.6 & 0.9 \\
BC & Mean & SD & Skewness & Kurtosis & Minimum & $25 \%$ Percentile & Median & $75 \%$ Percentile \\
BASAL & 1.7 & 0.5 & 2.1 & 11.9 & 0.3 & 1.3 & 0.5 & 1.6 \\
Mannitol & 0.9 & 0.4 & 2.9 & 19.4 & 0.1 & 0.1 & 0.8 & 1.1 \\
Stimulation & 0.5 & 0.4 & 2.7 & 21.2 & 0.009 & & 0.5 & 0.7 \\
\hline
\end{tabular}

indicated by white arrows in cortical areas as well as strong activation of the thalamus (red arrow) in this representative example. Cortical laminae were not visually observed in all the rats. The barrel cortex was, therefore, split into three distinct segments as performed by Herman et al. (2013) corresponding to cortical layers I-III, IV-V, and V-VI (Fig. 6b). $\mathrm{T}_{1}$ histograms of each segment were obtained after mannitol and TGN stimulation steps showing similar $\mathrm{T}_{1}$ shifts and similar shapes across segments (Fig. 6c) but an increased percentage of pixels in the segment corresponding to layer $\mathrm{V}-\mathrm{VI}$. The largest change in $\mathrm{T}_{1}$ after stimulation was observed in layer V-VI $(p \leq 0.007$, ANOVA) (Fig. 6d) for all the rats. The lowest $T_{1}$ values were also found in these cortical layers that contained the largest proportion of pixels with $\mathrm{T}_{1}$ values below $750 \mathrm{~ms}$ (70 \% in layer V-VI; $68 \%$ in layer IV-V; $63 \%$ in layer IIII) and no pixels with $\mathrm{T}_{1}$ above $1.35 \mathrm{~s}$.

\section{Discussion}

In the present work, AIM-MRI was successfully implemented at $9.4 \mathrm{~T}$ allowing $\mathrm{T}_{1}$ mapping and indirect mapping of calcium-dependent synaptic activation within the barrel cortex of 10 rats. Although the technique has demonstrated potential in the past (Aoki et al. 2002, 2004; Duong et al. 2000), it has never been fully exploited quantitatively. As shown in Tables 1 and 2, the number of animals and survival rate was always low for high $\mathrm{MnCl}_{2}$ concentrations although comparisons across studies were made difficult due to different anesthesia protocols. Here, a careful follow-up of heart rate and blood pressure during infusions of both mannitol and $\mathrm{MnCl}_{2}$ at $25 \mathrm{mM}$ helped preserving the rat's physiology. As soon as heart rate dropped significantly $(>10 \%)$, the infusion of $\mathrm{MnCl}_{2}$ was stopped and subsequently the rate of infusion was decreased. $\mathrm{MnCl}_{2}$ infusion could be continued after a few minutes upon recovery of heart rate and blood pressure to physiological values. The maintenance of a stable heart rate appears critical for an increased survival of animals.
Pixel-by-pixel $\mathrm{T}_{1}$ mapping was performed before infusion of $\mathrm{MnCl}_{2}$, after mannitol infusion in the internal carotid artery that induced BBB breakage of the left brain hemisphere and leakage of $\mathrm{MnCl}_{2}$ and finally after TGN stimulation. Upon stimulation, $\mathrm{Mn}^{2+}$ entered voltage-sensitive calcium channels and accumulated in the barrel cortex and in thalamic nuclei relaying inputs from the whiskers to the barrel cortex. The accumulation of paramagnetic $\mathrm{Mn}^{2+}$ in these specific areas induced a significant decrease of $\mathrm{T}_{1}$ compared to the stage where $\mathrm{Mn}^{2+}$ accumulated due to $\mathrm{BBB}$ breakage. Mean $\mathrm{T}_{1}$ values in the primary somatosensory cortex were in the same range $(0.63 \pm 0.12-1.9 \pm 0.3 \mathrm{~s})$ as those found in other studies (0.8-2.2 s) (Duong et al. 2000; Weng et al. 2007). In the thalamus, mean $T_{1}$ values were in the same range as in S1BF. In previous AIM-MRI experiments in the rat, $\mathrm{T}_{1}$ values in the thalamus were not reported and no contrast changes were visualized. However, Silva et al. (2008) and Tucciarone et al. (2009) showed strong enhancement of thalamo-cortical pathway and in particular of the layer IV in the primary somatosensory cortex following intracranial injection of $\mathrm{MnCl}_{2}$ in the thalamus. The patterns observed in our images (Fig. 6a) demonstrated strong similarities with theirs although our observations were made after a shorter period of time and after a stimulation period. Interestingly, we were able to observe cortical laminae in some rats in conjunction with strong activation of the thalamus or strong $\mathrm{Mn}^{2+}$ accumulation within the thalamus as was the case with intracranial injections in thalamic nuclei (Silva et al. 2008; Tucciarone et al. 2009).

Histogram distributions offered a visualization of the shift in $\mathrm{T}_{1}$ toward lower values after mannitol injection and after TGN stimulation in the barrel cortex and in the thalamic nuclei. $\mathrm{T}_{1}$ values were decreased down to $50-350 \mathrm{~ms}$ with a significant increase of the frequency of pixels in this range of values. The percentage of pixels in this range of values corresponded exactly to the percentage of pixels that had shifted due to stimulation only. In other words, histogram analysis was able to demonstrate that defined proportions of the barrel cortex and the thalamus were 

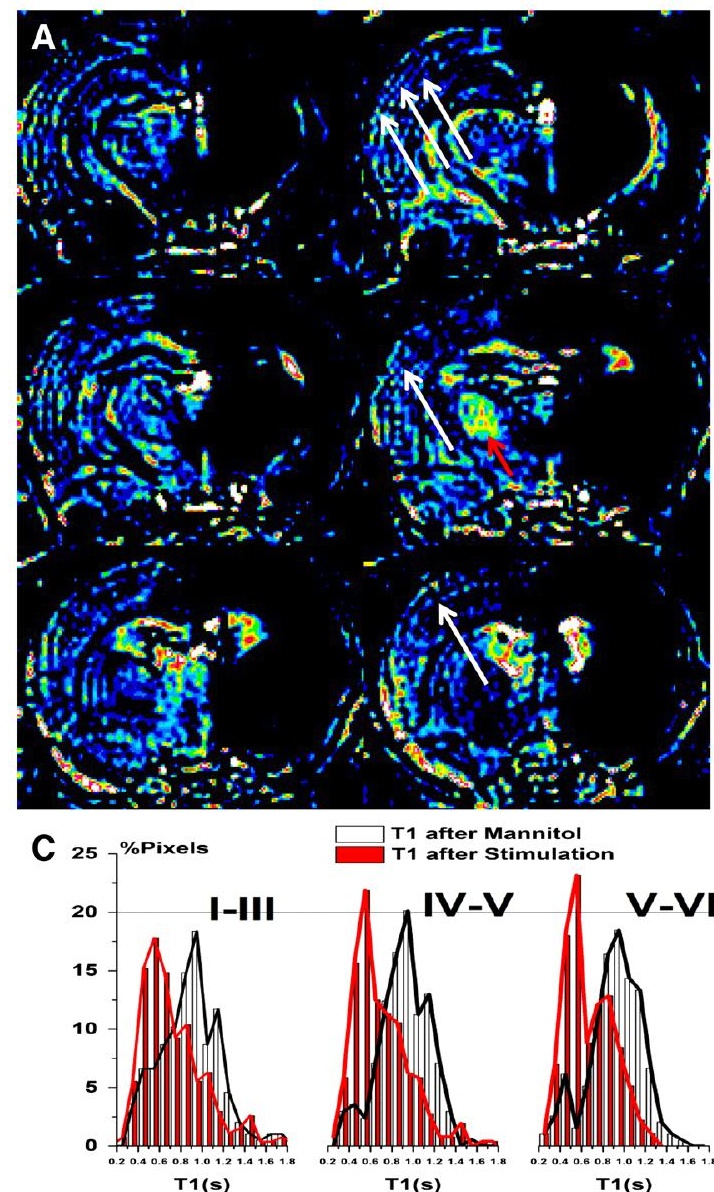

$\square 1$ after Mannitol
$\square$ T1 after Stimulation
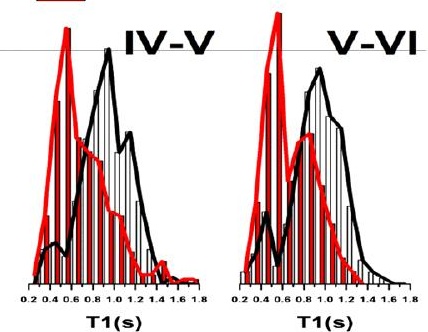

Fig. 6 a Difference images obtained after subtracting successive 3D T1-weighted GRE images after TGN Stimulation and 3D T1weighted GRE images post-mannitol infusion. Cortical layers could be depicted as indicated by arrows. b The barrel cortex was split into three distinct segments corresponding to cortical layers I-III, IV-V, and V-VI as shown on an axial slice extracted from the Paxinos atlas

sensitive to depolarization of voltage-sensitive $\mathrm{Ca}^{2+}$ channels due to TGN stimulation solely.

One of the disadvantages of histogram analysis is the lack of spatial correspondence to the pixel-by-pixel mapping. In addition due to the importance of performing relatively rapid MRI experiments to conserve adequate physiological conditions for rats in the magnet, high-resolution MR images were not acquired in the present study in contrast to studies by Silva et al. (2008) or Tucciarone et al. (2009). Here, the investigation of distributions of pixels allowed characterizing different populations of pixels in low resolution images. For this reason and to examine the distribution of $\mathrm{T}_{1}$ values in cortical layers, we investigated the barrel cortex by dividing it into three segments of identical size as performed by Herman et al. (2013). The largest change in $\mathrm{T}_{1}$ was observed in the segment corresponding to layer V-VI. To date, it was shown that MEMRI
B
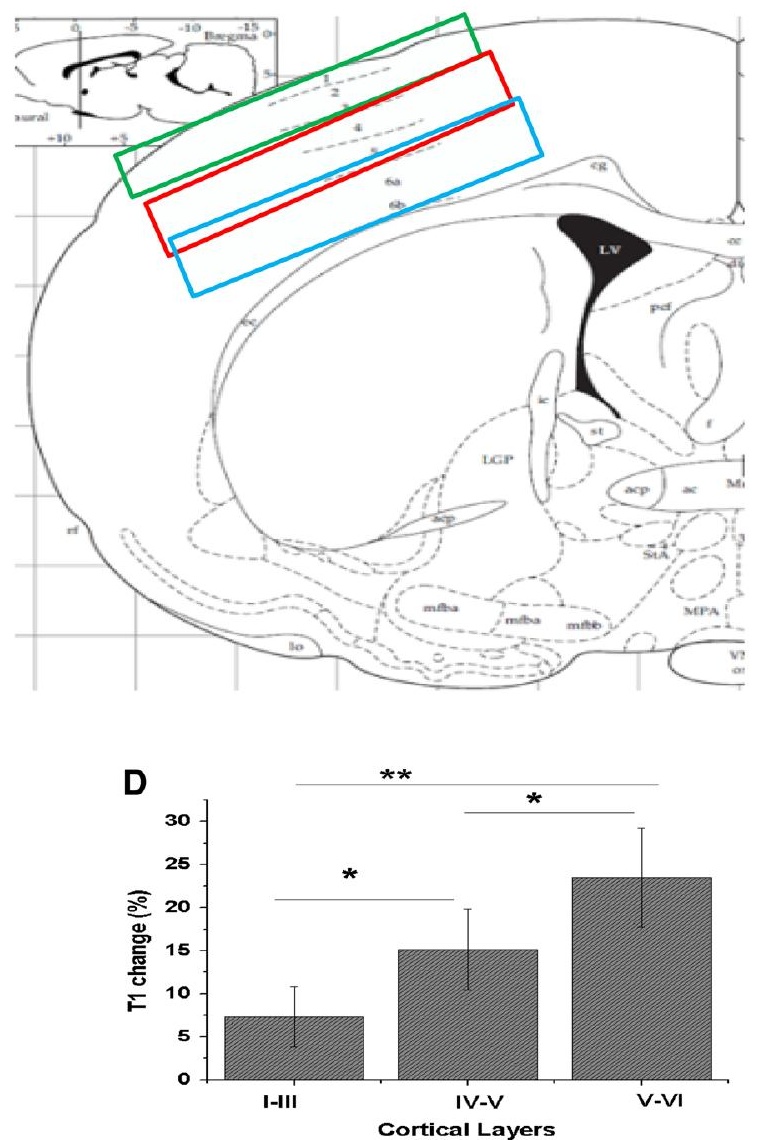

and as performed by Herman et al. (2013). c $\mathrm{T}_{1}$ histograms of each segment were obtained after mannitol and TGN stimulation steps showing similar $T_{1}$ shifts and similar shapes across segments but a slightly higher percentage of pixels with lower $\mathrm{T}_{1} \mathrm{~s}$ in segment 3 and following TGN stimulation. d The largest change in $\mathrm{T}_{1}$ after stimulation was observed in layer V-VI. $(* P<0.05 ; * * p \leq 0.007)$

can detect specific layers in cortical areas and that the activity-dependent properties of MEMRI were specific for localized cortical areas (Table 1). As pointed out by Herman et al. (2013), it is of paramount importance to understand functional MRI signals across laminae in terms of neural activity since quantitative data on layer-specific variations are lacking. In the present study, we were able to detect cortical cytoarchitecture during a functional paradigm extending the potential of AIM-MRI. In their study, Herman et al. (2013) showed a decrease of BOLD and cerebral blood volume responses in the rat somatosensory cortex from upper superficial to deep layers. On the other hand, multi-unit spike activity (MUA) and cerebral metabolic rate of oxygen $\left(\mathrm{CMRO}_{2}\right)$ increased. It is interesting to note that the relative changes in $\mathrm{T}_{1}$ due to TGN stimulation increased from the superficial layers to the deep layers in the same manner as for the MUA and $\mathrm{CMRO}_{2}$ laminar 
responses. These similarities further comfort us that $T_{1}$ changes due to $\mathrm{Mn}^{2+}$ accumulation in the primary somatosensory cortex following stimulation reflect neuronal activity. Herman et al. (2013) also showed that the somatosensory cortex responses acquired with various modalities were well correlated between layers (high Pearson's $r$ score) and in particular middle and lower segments had similar responses with MUA as indicated by a slope of 1 . In the present study, $T_{1}$ values between segments after the TGN stimulation stage were also well correlated in the range $0.2-1 \mathrm{~s}$ with slopes close to 1.0 for all the layers (not shown). Understanding how neural activity is distributed among cortical layers in the barrel cortex is complex (Herman et al. 2013; Yu et al. 2014). Recently, Yu et al. (2014) concluded that the location of BOLD fMRI onset matches well with the input layers across the cortical depth as detected by MEMRI. However, they also mentioned that differences in timing between fMRI and neural events make their results difficult to interpret.

Cortical layers of the somatosensory cortex represent different cell types: layer IV spiny stellate neurons receive whisker-specific synaptic inputs from the thalamic VPM and relay excitation to other cortical layers. Layer $\mathrm{V}$ pyramidal cells on the other hand receive robust thalamocortical inputs at basal dendrites through which action potentials (APs) back propagate. Although supporting data will be needed in the future, kurtosis and skewness parameters as well as differences between percentiles in $T_{1}$ distributions may reflect the different cell types and energetic needs associated with different functions of the six cortical layers. Thus, AIM-MRI combined to histogram analysis may allow in-depth characterization of cortical layers during brain activity without the need for timeconsuming acquisitions of high-resolution MR images. Other techniques are being implemented in our laboratory for an increased spatial resolution of MEMRI sequences allowing an increased anatomical depiction of cortical layers (Marques and Gruetter 2013). In the present study, instead of using MEMRI images for structural localization only (Tucciarone et al. 2009; Yu et al. 2013) requiring several hours of acquisition, AIM-MRI allowed indirect quantitative assessment of neural activity simultaneously in different layers through the indirect quantitative assessment of calcium-dependent synaptic activity. Calciumdependent synaptic activity is usually estimated by fluorescence microscopy (Nuñez et al. 2012) and usually focuses on a single layer only (Nuñez et al. 2012). Although spatially specific, these methodologies have demonstrated that the generation and propagation of $\mathrm{Ca}^{2+}$ transients in the somatosensory cortex are complex and dependent on various factors such as glutamatergic transmission (Nevian and Sakmann 2004), cholinergic innervations (Nuñez et al. 2012; Lauritzen 2005) themselves dependent on activation of NMDA receptors, nicotinic cholinergic and muscarinic receptors. Altogether these factors have an impact on $\mathrm{Ca}^{2+}$ spikes that modify the excitatory/inhibitory balance within and across layers. Moreover, several studies (Schulz et al. 2012; Nuñez et al. 2012; Lauritzen 2005) have shown that $\mathrm{Ca}^{2+}$ spikes have a key role to play in plastic events and in maintaining sustained stimulation. The stimulation of barrel cortex in rats showed for example that acetylcholine increased excitability and excitatory transmission and decreased synaptic inhibition in the cortical layer $\mathrm{V}$. These mechanisms facilitated the generation of $\mathrm{Ca}^{2+}$ spikes mediated by voltage-gated $\mathrm{Ca}^{2+}$ channels in layer $\mathrm{V}$ of the barrel cortex (Nuñez et al. 2012). Functional MR spectroscopic techniques have recently been translated to animals (Just et al. 2013) and have demonstrated significant neurochemical changes (Glutamate and Lactate) in the rat barrel cortex during activation in accordance with previous results in the human cortex. We, therefore, truly believe that indirect mapping of calcium-dependent synaptic activity in cortical layers with quantitative AIM-MRI could help interpreting neurovascular and neurometabolic couplings in the cerebral cortex.

Other studies quantified the firing rates of barrel cortex neurons across cortical layers. Firing rates were significantly different and were the highest in layer IV and V of the barrel cortex but only a small fraction of the neurons fired the majority of spikes (O'Connor et al. 2010). The increased changes in $T_{1}$ seen in layer V-VI (lower segment) due to TGN stimulation in the present study and in neural activity (MUA) in the study performed by Herman et al. (2013) may, therefore, reflect differences in spiking activity between layers giving rise to increased $\mathrm{Ca}^{2+}$ transients due to various mediators that as a consequence induce plastic changes as well as long-term potentiation or depression or adaptation processes. Several recent studies have used high-resolution BOLD fMRI to investigate neurovascular coupling issues in cortical layers of the rat primary somatosensory cortex (Herman et al. 2013; Shih et al. 2013; Yu et al. 2014). Because of the different neuronal populations in these cortical layers, it was expected to obtain an improved interpretation of the underlying neural activity. The general conclusion from these studies was that local neural activity cannot be entirely characterized by hemodynamic responses. By contrast, MEMRI techniques have shown in the past a very accurate description of cortical structures. Here, AIM-MRI data presented interesting similarities with local neural activity. Moreover, in discussions about neurovascular coupling, the role of $\mathrm{Ca}^{2+}$ fluxes has been neglected although calcium signals have been related to BOLD signals in the primary somatosensory cortex with specific identification of cell types (Schulz et al. 2012). 


\section{Conclusions}

The present study evaluated the potential of activityinduced manganese-dependent MRI quantitative data in the primary somatosensory barrel cortex and the thalamus of the rat. Although toxicity of manganese and mannitol and opening of the BBB remain difficult, the quantitative outcome of AIM-MRI using pixel-by-pixel $\mathrm{T}_{1}$ data analyzed with histogram distributions yielded increased knowledge of barrel cortex and thalamus during activations independent of hemodynamic responses. In particular, cortical layers were analyzed showing increased responses in deeper layers of the primary somatosensory cortex in accordance with previous literature results. Thus, $\mathrm{T}_{1}$ data following accumulation of manganese in activated regions of the brain represent good surrogate markers of local calcium-dependent synaptic activity. AIM-MRI could offer a means of quantitatively interpreting neural activity simultaneously across several cortical layers while identification of the role of calcium dynamics in vivo during brain activation could play a key role in resolving neurovascular coupling mechanisms.

Acknowledgments This study was supported by the Centre d'Imagerie BioMédicale (CIBM) of Ecole Polytechnique Fédérale de Lausanne (EPFL), the University of Lausanne (UNIL) and the Foundations Leenards et Jeantet. The authors would like to acknowledge strong technical support from Dr Mario Lepore and Hanne Frenkel from the CIBM veterinary team.

Conflict of interest The authors have no conflict of interest to disclose.

\section{References}

Aoki I, Tanaka C, Takegami T, Ebisu T, Umeda M, Fukunaga M, Fukuda K, Silva AC, Koretsky AP, Naruse S (2002) Dynamic activity-induced manganese-dependent contrast magnetic resonance imaging (DAIM MRI). Magn Reson Med 48(6):927-933

Aoki I, Naruse S, Tanaka C (2004) Manganese-enhanced magnetic resonance imaging (MEMRI) of brain activity and applications to early detection of brain ischemia. NMR Biomed 17(8):569-580

Benedetti B, Matyash V, Kettenmann H (2011) Astrocytes control GABAergic inhibition of neurons in the mouse barrel cortex. J Physiol 589(Pt 5):1159-1172

Chuang KH, Lee JH, Silva AC, Belluscio L, Koretsky AP (2009) Manganese enhanced MRI reveals functional circuitry in response to odorant stimuli. Neuroimage 44(2):363-372

Duong TQ, Silva AC, Lee SP, Kim SG (2000) Functional MRI of calcium-dependent synaptic activity: cross correlation with CBF and BOLD measurements. Magn Reson Med 43(3):383-392

Egger V, Feldmeyer D, Sakmann B (1999) Coincidence detection and changes of synaptic efficacy in spiny stellate neurons in rat barrel cortex. Nat Neurosci 2(12):1098-1105

Fa Z, Zhang R, Li P, Zhang J, Zhang P, Zhu S, Wu Q, Huang F, Liu Y, Yang L, Chang H, Wen Z, Gao D, Zeng Y, Jiang X (2011) Effects of temporarily disrupting BBB on activity-induced manganese-dependent functional MRI. Brain Imaging Behav 5(3): 181-188

Helmchen F, Svoboda K, Denk W, Tank DW (1999) In vivo dendritic calcium dynamics in deep-layer cortical pyramidal neurons. Nat Neurosci 2(11):989-996

Herman P, Sanganahalli BG, Blumenfeld H, Rothman DL, Hyder F (2013) Quantitative basis for neuroimaging of cortical laminae with calibrated functional MRI. Proc Natl Acad Sci U S A. 110(37):15115-15120

Inoue T, Majid T, Pautler RG (2011) Manganese enhanced MRI (MEMRI): neurophysiological applications. Rev Neurosci 22(6):675-694

Just N, Gruetter R (2011) Detection of neuronal activity and metabolism in a model of dehydration-induced anorexia in rats at $14.1 \mathrm{~T}$ using manganese-enhanced MRI and 1H MRS. NMR Biomed 24(10):1326-1336

Just N, Petersen C, Gruetter R (2010) BOLD responses to trigeminal nerve stimulation. Magn Reson Imaging 28(8):1143-1151

Just N, Cudalbu C, Lei H, Gruetter R (2011) Effect of manganese chloride on the neurochemical profile of the rat hypothalamus. J Cereb Blood Flow Metab 31(12):2324-2333

Just N, Xin L, Frenkel H, Gruetter R (2013) Characterization of sustained BOLD activation in the rat barrel cortex and neurochemical consequences. Neuroimage 74:343-351

Koretsky AP, Silva AC (2004) Manganese-enhanced magnetic resonance imaging (MEMRI). NMR Biomed 17(8):527-531

Lauritzen M (2005) Reading vascular changes in brain imaging: is dendritic calcium the key? Nat Rev Neurosci 6(1):77-85

Lecrux C, Toussay X, Kocharyan A, Fernandes P, Neupane S, Lévesque M, Plaisier F, Shmuel A, Cauli B (2011) Hamel E Pyramidal neurons are "neurogenic hubs" in the neurovascular coupling response to whisker stimulation. J Neurosci 31(27):9836-9847

Lin YJ, Koretsky AP (1997) Manganese ion enhances T1-weighted MRI during brain activation: an approach to direct imaging of brain function. Magn Reson Med 38(3):378-388

Lu H, Patel S, Luo F, Li SJ, Hillard CJ, Ward BD, Hyde JS (2004) Spatial correlations of laminar BOLD and CBV responses to rat whisker stimulation with neuronal activity localized by Fos expression. Magn Reson Med 52(5):1060-1068

Marques JP, Gruetter R (2013) New developments and applications of the MP2RAGE sequence-focusing the contrast and high spatial resolution R1 mapping. PLoS One 8(7):e69294

Mlynárik V, Gambarota G, Frenkel H, Gruetter R (2006) Localized short-echo-time proton MR spectroscopy with full signal-intensity acquisition. Magn Reson Med 56(5):965-970

Nevian T, Sakmann B (2004) Single spine Ca2 + signals evoked by coincident EPSPs and backpropagating action potentials in spiny stellate cells of layer 4 in the juvenile rat somatosensory barrel cortex. J Neurosci 24(7):1689-1699

Norup Nielsen A, Lauritzen M (2001) Coupling and uncoupling of activity-dependent increases of neuronal activity and blood flow in rat somatosensory cortex. J Physiol 533(Pt 3):773-785

Nuñez A, Domínguez S, Buño W, Fernández de Sevilla D (2012) Cholinergic-mediated response enhancement in barrel cortex layer V pyramidal neurons. J Neurophysiol 108(6):1656-1668

O'Connor DH, Peron SP, Huber D, Svoboda K (2010) Neural activity in barrel cortex underlying vibrissa-based object localization in mice. Neuron 67(6):1048-1061

Parker GJ, Baustert I, Tanner SF, Leach MO (2000) Improving image quality and $\mathrm{T}(1)$ measurements using saturation recovery turboFLASH with an approximate K-space normalisation filter. Magn Reson Imaging 18(2):157-167

Pautler RG (2006) Biological applications of manganese-enhanced magnetic resonance imaging. Methods Mol Med 124:365-386 
Paxinos G, Watson C (1998) The rat brain in stereotaxic coordinates. Academic Press, San Diego

Schulz K, Sydekum E, Krueppel R, Engelbrecht CJ, Schlegel F, Schröter A, Rudin M, Helmchen F (2012) Simultaneous BOLD fMRI and fiber-optic calcium recording in rat neocortex. Nat Methods 9(6):597-602

Serrano F, Deshazer M, Smith KD, Ananta JS, Wilson LJ, Pautler RG (2008) Assessing transneuronal dysfunction utilizing manganese-enhanced MRI (MEMRI). Magn Reson Med 60(1): 169-175

Shih YY, Chen YY, Lai HY, Kao YC, Shyu BC, Duong TQ (2013) Ultra high-resolution fMRI and electrophysiology of the rat primary somatosensory cortex. Neuroimage 73:113-120

Silva AC, Lee JH, Wu CW, Tucciarone J, Pelled G, Aoki I, Koretsky AP (2008) Detection of cortical laminar architecture using manganese-enhanced MRI. J Neurosci Methods 167(2):246-257

Stosiek C, Garaschuk O, Holthoff K, Konnerth A (2003) In vivo twophoton calcium imaging of neuronal networks. Proc Natl Acad Sci USA 100(12):7319-7324

Svoboda K, Helmchen F, Denk W, Tank DW (1999) Spread of dendritic excitation in layer $2 / 3$ pyramidal neurons in rat barrel cortex in vivo. Nat Neurosci 2(1):65-73

Tucciarone J, Chuang KH, Dodd SJ, Silva A, Pelled G, Koretsky AP (2009) Layer specific tracing of corticocortical and thalamocortical connectivity in the rodent using manganese enhanced MRI. Neuroimage 44:923-931
Van der Linden A, Van Meir V, Tindemans I, Verhoye M, Balthazart J (2004) Applications of manganese-enhanced magnetic resonance imaging (MEMRI) to image brain plasticity in song birds. NMR Biomed 17(8):602-612

Wang X, Lou N, Xu Q, Tian GF, Peng WG, Han X, Kang J, Takano T, Nedergaard M (2006) Astrocytic $\mathrm{Ca}^{2+}$ signaling evoked by sensory stimulation in vivo. Nat Neurosci 9(6):816-823

Weng JC, Chen JH, Yang PF, Tseng WY (2007) Functional mapping of rat barrel activation following whisker stimulation using activity-induced manganese-dependent contrast. Neuroimage 36(4):1179-1188

Yu X, Chung S, Chen DY, Wang S, Dodd SJ, Walters JR, Isaac JT, Koretsky AP (2012a) Thalamocortical inputs show post-criticalperiod plasticity. Neuron 74(4):731-742

Yu X, Glen D, Wang S, Dodd S, Hirano Y, Saad Z, Reynolds R, Silva AC (2012b) Koretsky AP Direct imaging of macrovascular and microvascular contributions to BOLD fMRI in layers IV-V of the rat whisker-barrel cortex. Neuroimage 59(2):1451-1460

Yu X, Qian C, Chen DY, Dodd SJ, Koretsky AP (2014) Deciphering laminar-specific neural inputs with line-scanning fMRI. Nat Methods 11(1):55-58

Zhang R, Fa Z, Liu Y, Sun H, Li P, Li S, Wang X, Lei H, Jiang X (2014) Dynamic MRI of rat brain following manganese administration through the internal carotid artery. Neurol Res 36(7):679-686. doi:10.1179/1743132813Y.0000000291 